\title{
RECOGNIZING $\sigma$-MANIFOLDS
}

\author{
JAMES P. HENDERSON
}

\begin{abstract}
Denote by $\sigma$ the subspace of the Hilbert cube consisting of $\left\{\left(x_{i}\right): x_{i}=\mathbf{0}\right.$ for all but finitely many $\left.i\right\}$. Then following characterization of manifolds modeled on $\sigma$ is proven and applied to cell-like, upper semicontinuous decompositions of $\sigma$-manifolds. An ANR $X$ is a $\sigma$-manifold if and only if (a) $X$ is the countable union of finite-dimensional compacta, (b) each compact subset of $X$ is a strong $Z$-set, and (c) for each positive integer $k$, every mapping $f: R^{k} \rightarrow X$ can be arbitrarily closely approximated by an injection.
\end{abstract}

1. Introduction. One of the major problems in topology is that of characterizing a given class of topological spaces. The problem of characterizing topological manifolds has a long, involved history [3]. Two of the more recent characterizations are those of Torunczyk [13] for Hilbert cube manifolds and the combined works of Edwards [7] and Quinn [11] for $n$-manifolds, $n \geq 5$. These characterizations each contain a short list of properties which are reasonably easy to verify.

The manifolds to be discussed in this paper are those manifolds modeled on $\sigma$, where $\sigma$ is the subspace of the Hilbert cube consisting of $\left\{\left(x_{i}\right): x_{i}=0\right.$ for all but finitely many $i\}$. There have been several characterizations of $\sigma$ given in the past $[\mathbf{2}, \mathbf{1 2}, \mathbf{1 5}]$, all somewhat difficult to apply due to the necessity of identifying certain structures in the manifold candidate $X$. More recently, Mogilski $[\mathbf{1 0}]$ had given a characterization of $\sigma$-manifolds. However, due to an example constructed by P. Bowers, an error in an earlier result of Torunczyk upon which Mogilski had based his work was detected. Thus Mogilski has slightly modified his result in [10], and the new characterization is

An ANR $X$ is a $\sigma$-manifold if and only if

(a) $X$ is the countable union of finite-dimensional compacta,

(b) each compact subset of $X$ is a strong $Z$-set,

(c) each mapping $f: A \rightarrow X$ from a finite-dimensional compacta into $X$ that restricts to an embedding on a closed subset $B$ of $A$ can be arbitrarily closely approximated by an embedding extending the rstriction $f \mid B$.

This characterization is better in some sense than the previous ones in that it relies partially on the verification of a certain kind of general position rather than solely the internal structure conditions of its predecessors. However, condition (c) is somewhat awkward in that the finite-dimensional compactum $A$ has little structure with which to work. The first result of this paper is the following characterization of $\sigma$-manifolds.

Received by the editors July 26, 1984 and, in revised form, October 12, 1984.

1980 Mathematics Subject Classification. Primary 57N20, 58B05; Secondary 54B15, 54C99.

Key words and phrases. Countable dimensional manifold, cell-like decompositions, Euclidean injection property. 
THEOREM. An ANR $X$ is a $\sigma$-manifold provided

(a) $X$ is the countable union of finite-dimensional compacta,

(b) each compact subset of $X$ is a strong $Z$-set,

(c) each mapping $f: R^{k} \rightarrow X$ can be arbitrarily closely approximated by an injection for any positive integer $k$.

The above Theorem allows one to deal with mappings of Euclidean spaces into the space $X$ instead of mappings of pairs of finite-dimensional compacta, but still with the internal condition concerning strong $Z$-sets. However, our interest in this Theorem is in its applications to decompositions of $\sigma$-manifolds. If $G$ is a cell-like, upper semicontinuous decomposition of a $\sigma$-manifold $M$, each compact subset of $M / G$ will be a strong $Z$-set. Thus, when applying the Theorem to $M / G$, one need only verify conditions (a) and (c). Also, it is a straightforward matter to lift mappings $f$ of $R^{k}$ into $M / G$ to mappings of $R^{k}$ into $M[9]$, allowing one to possibly use the structure of $M$ and $R^{k}$ to improve the mapping $f$. So when trying to identify those decomposition spaces which are $\sigma$-manifolds, the Theorem provides an improvement over earlier results. This will be illustrated in the applications which are given in the last section.

Finally, the author would like to thank Doug Curtis for suggesting several changes and corrections on an earlier version of this paper.

2. Preliminaries. All spaces will be separable metric spaces. Viewing the Hilbert cube $Q$ as $\prod_{i=1}^{\infty}[-1,1], \sigma$ is $\left\{\left(x_{i}\right): x_{i}=0\right.$ for all but finitely many $\left.i\right\}$. A $\sigma$-manifold is a space having an open cover of sets each homeomorphic to some open subset of $\sigma$. If $M_{1}$ and $M_{2}$ are $\sigma$-manifolds of the same homotopy type, then $M_{1}$ and $M_{2}$ are homeomorphic to [5].

A closed subset $A$ of an ANR $X$ is a $Z$-set provided for each $\delta: X \rightarrow(0,1)$, there is a mapping $h: X \rightarrow X \backslash A$ with $d(x, h(x))<\delta(x)$, where $d$ is the metric on $X$. If, in addition, one can always choose $h$ so that the closure of $h(X)$ in $X$ misses $A$, then $A$ is a strong $Z$-set. Each compact subset $A$ of a $\sigma$-manifold $M$ is a strong $Z$-set.

The space $X$ is said to be strongly universal for finite-dimensional compacta if each mapping $f: A \rightarrow X$ from a finite-dimensional compacta into $X$ that restricts to an embedding on a closed subset $B$ of $A$ can be arbitrarily closely approxi_nated by an embedding extending the restriction $f \mid B$. Note that this is Mogilski's condition (c).

A set $X$ is said to satisfy the Euclidean injection property (EIP) if for each Euclidean space $R^{k}, k \geq 1, \delta: R^{k} \rightarrow(0,1)$, and mapping $f: R^{k} \rightarrow X$, there is an injection, that is, a one-to-one mapping, $f^{\prime}: R^{k} \rightarrow X$ with $d\left(f(x), f^{\prime}(x)\right)<$ $\delta(x)$. Note that if $X$ satisfies the EIP, then $X$ must be at least countably infinite dimensional, and that both $\sigma$ and $Q$ satisfy the EIP.

A compact space $A$ is cell-like provided it has the shape of a point. An upper semicontinuous (u.s.c.) decomposition $G$ of a space is cell-like provided each element $g \in G$ is cell-like. If $G$ is a cell-like, u.s.c. decomposition of a $\sigma$-manifold $M$, and $M / G$ is an ANR, then the decomposition mapping is a fine homotopy equivalence, and it follows that each compact subset of $M / G$ is also a strong $Z$-set. 
3. Characterizing $\sigma$-manifolds. In reviewing Mogilski's characterization, it seemed that the condition involving approximating mappings of pairs of finitedimensional compacta would most easily lend itself to simplification. Certainly at this time the techniques for verifying that a space is countable dimensional, much less the countable union of finite-dimensional compacta, are cumbersome at best. In the special cases considered in the applications, the condition that the space be an ANR will follow from the countable dimensionality requirements and need not be hypothesized. However, in general, one must require ihat the space be an ANR.

The choice of replacing the finite-dimensional pairs by Euclidean spaces was influenced by the work of Cannon [4], Edwards [7] and Torunczyk [13] as well as the need for injected images of noncompact, finite-dimensional metric spaces. Mogilski's mapping criterion essentially requires that images of finite-dimensional, $\sigma$-compact metric spaces be arbitrarily closely approximable by injections. Since the Euclidean spaces are the basic models of such spaces, the Euclidean injection property (EIP) was the natural choice.

We begin with a lemma which illustrates the connection between the EIP and the ability to obtain one-to-one approximations of mappings of open subsets of $R^{k}$ into an ANR.

LEMMA 3.1. Let $X$ be an ANR with EIP and the property that every finitedimensional compact subset of $X$ is a $Z$-set. Then for each open subset $U$ of $R^{k}$, mapping $f: U \rightarrow X$, and $\delta: U \rightarrow(0,1)$, there is an injection $g: U \rightarrow X$ which $\delta$-approximates $f$.

ProOF. Let $S=\left\{S_{1}, S_{2}, \ldots, S_{n}, \ldots\right\}$ be the $k$-simplices of a triangulation of $U$. For each $k$-simplex $S_{n}$, we may choose an open set $U_{n}$ in $U$ such that

(1) $U_{n} \cong R^{k}$

(2) $U_{n} \supseteq S_{n}-\bigcup_{i=1}^{n-1} S_{i}$,

(3) $U_{n} \cap S_{i}=\emptyset$ iff $S_{1} \cap S_{n}=\emptyset$ or $i<n$.

Note that $U=\bigcup_{i=1}^{\infty} U_{i}$.

The EIP implies the existence of a mapping $f_{1}: U \rightarrow X$ satisfying

(1) $f_{1} \mid U_{1}$ is an injection,

(2) $f_{1} \mid U-U_{1}=f_{\mid} U-U_{1}$,

(3) $d\left(f(x), f_{1}(x)\right)<\delta(x) / 2 k$ for $x \in S_{k}, k$ is a positive integer.

The new mapping $f_{1}$ will be an embedding when restricted to $S_{1}$, but may not be one-to-one over $f_{1}\left(S_{1}\right)$. Since the compact subset $f_{1}\left(S_{1}\right)$ is a $Z$-set, standard techniques may be employed to obtain a mapping $g_{1}: U \rightarrow X$ with

(1) $g_{1}\left|S_{1}=f_{1}\right| S_{1}$

(2) $g_{1}\left(U-S_{1}\right) \cap g_{1}\left(S_{1}\right)=\emptyset$,

(3) $d\left(g_{1}(x), f_{1}(x)\right)<\delta(x) / 2 k$ for $x \in S_{k}, k$ is a positive integer.

Now $g_{1}$ is one-to-one over $g_{1}\left(S_{1}\right)$. This provides the initial function for the recursive definition of the mappings which will be used to construct $g$. For $n>1$, use the EIP to define $f_{n}: U \rightarrow X$ subject to

(1) $f_{n} \mid U_{n}$ is an injection,

(2) $f_{n}$ and $g_{n-1}$ agree on $U-U_{n}$,

(3) $d\left(f_{n}(x), g_{n-1}(x)\right)<\delta(x) / 2 k$ for $x \in S_{k}, k$ a positive integer,

(4) $f_{n}\left(U_{n}\right) \cap g_{n-1}\left(\bigcup_{i=1}^{n-1} S_{i}\right)=\emptyset$. 
Next, move $f_{n}\left(U-\bigcup_{i=1}^{n} S_{i}\right)$ off of $f_{n}\left(\bigcup_{i=1}^{n} S_{i}\right)$ via the mapping $g_{n}: U \rightarrow X$ where

(1) $g_{n}\left|\bigcup_{i=1}^{n} S_{i}=f_{n}\right| \bigcup_{i=1}^{n} S_{i}$

(2) $g_{n}\left(U-\bigcup_{i=1}^{n} S_{i}\right) \cap g_{n}\left(\bigcup_{i=1}^{n} S_{i}\right)=\emptyset$,

(3) $d\left(g_{n}(x), f_{n}(x)\right)<\delta(x) / 2 k$ for $x \in S_{k}, k$ a positive integer.

We may now define $g(x)=\lim g_{n}(x)=g_{k}(x)$, where $x \in S_{k}$. Clearly, $g$ is an injection. Also, for $x \in P$, there is a smallest integer $n$ such that $x \in S_{n}$. Then if we let $f=g_{0}$,

$$
d(f(x), g(x))=d\left(g_{0}(x), g_{n}(x)\right) \leq \sum d\left(g_{i-1}(x), g_{i}(x)\right)<n(\delta(x) / n)=\delta(x) .
$$

Thus $g$ is the desired one-to-one mapping.

At this point, we turn to the relationship between the EIP and strong universality for finite-dimensional compacta.

PROPOSITION 3.2. An ANR $X$ is strongly universal for finite-dimensional compacta if and only if

(a) each finite-dimensional compact subset of $X$ is a $Z$-set,

(b) $X$ satisfies the EIP.

ProOF. First, assume that $X$ is strongly universal for finite-dimensional compacta. It was shown in [10] that (a) holds, so it remains to verify (b). Let $f: R^{k} \rightarrow X$ and $\delta: R^{k} \rightarrow(0,1)$ be given. Denote by $B(n)$ the $k$-ball in $R^{k}$ of radius $n$ centered at the origin, $n$ a nonnegative integer, and let $\delta_{n}=\min \{\delta(x): x \in B(n)\}$. By labeling $f=f_{0}$, one may use the hypothesis and the fact that $X$ is an ANR to inductively obtain mappings $f_{n}: R^{k} \rightarrow X, n \geq 1$, such that

(1) $f_{n}\left|B(n-1) \cup\left(R^{k}-B(n+1)\right)=f_{n-1}\right| B(n-1) \cup\left(R^{k}-B(n+1)\right)$,

(2) $f_{n} \mid B(n)$ is an embedding,

(3) $d\left(f_{n}(x), f_{n-1}(x)\right)<\delta_{n+1}$.

If $f^{\prime}(x)=\lim f_{n}(x)$, then $f^{\prime}$ is the desired injection.

Conversely, we now assume that $X$ satisfies conditions (a) and (b), and verify that the requirements of strong universality are met. Let $(A, B)$ be a pair of finitedimensional compacta. For $g: A \rightarrow X$ continuous with $g \mid B$ an embedding, it is necessary to construct an embedding $\tilde{g}: A \rightarrow X$ with $g|B=\tilde{g}| B$ and the mappings $g$ and $\tilde{g}$ being as close as desired.

Since $g(B)$ is a compact subset of $X$, condition (a) implies that $g(B)$ is a $Z$-set for $X$. This, along with the fact that $X$ is an ANR, allows one to assume that $g(B) \cap g(A-B)=\emptyset$. Therefore, the problem is reduced to showing that $g \mid A-B$ can be arbitrarily closely approximated by an injection $\tilde{g}: A-B \rightarrow X-g(B)$ which can be extended to $B$ by $g \mid B$.

By first embedding $A$ in the $R^{k-1}$ boundary of a half-plane $R_{+}^{k}$, and then pushing $A-B$ into $R_{+}^{k}-R^{k-1} \cong R^{k}$, we may assume that $A-B$ is embedded as a closed subset of $R^{k}$. In the simplest situation, we first assume that $X$ is an ANR. In this case, the mapping $g \mid A-B: A-B \rightarrow X$ be extended to all of $R^{k}$. Applying the EIP, this extension $f: R^{k} \rightarrow X$ may be approximated so closely by an injection $f^{\prime}: R^{k} \rightarrow X$ that $\tilde{g}=f^{\prime} \mid A-B$ is the required mapping.

The general case where $X$ is an ANR is treated in a similar manner. We may extend $g \mid A-B$ to an open subset $U$ of $R^{k}$. An application of Lemma 3.1 to the extension yields the desired result. 
The alternate characterization of $\sigma$-manifolds is now a direct result of Proposition 3.2. Since all compacta are assumed to be strong $Z$-sets, we may simply replace Mogilski's condition (c) with the EIP.

THEOREM 3.3. An ANR $X$ is a $\sigma$-manifold if and only if $X$ satisfies the following conditions:

(a) $X$ is the countable union of finite-dimensional compacta,

(b) each compact subset of $X$ is a strong $Z$-set,

(c) $X$ satisfies the EIP.

4. Applications to decomposition spaces. As pointed by Walsh [14], there are often two steps involved in showing that a space $X$ is a manifold. The first step is to obtain a resolution of a manifold $M$ onto $X$. The second is to show that given conditions on $X$ allows one to conclude that $M$ and $X$ are homeomorphic. In this section we will assume that a resolution exists by taking the space $X$ to be a cell-like, u.s.c. decomposition space of a $\sigma$-manifold $M$. Throughout, $M / G$ will be the decomposition space. Since $M / G$ will have the same homotopy type as $M$, it will follow from Chapman [5] that $M$ and $M / G$ will be homeomorphic if $M / G$ is a $\sigma$-manifold. Thus in each of the applications we will only verify that $M / G$ is a $\sigma$-manifold.

The first application was one motivation for proving the above Theorem. One would like to be able to replace condition (c) of Mogilski's characterization with something similar to the DDP. Recall that for an ANR $Y$ which is a complete metric space, $Y$ satisfies the DDP if and only if mappings of a 2-cell into $Y$ can be arbitrarily closely approximated by embeddings [4]. Our analogy is the EIP. The cell-like decomposition setting allows one to replace Mogilski's condition (c) with the EIP, and, in addition, drop the ANR hypothesis.

Proposition 4.1. If $M / G$ is the countable union of finite-dimensional compacta and satisfies the Euclidean injection property, then $M / G \cong M$.

Proof. Since $M / G$ is the countable union of finite-dimensional compacta, it is countable dimensional, and hence an ANR [1]. Standard lifting arguments [9] may be employed to show that compact subsets of $M / G$ are strong $Z$-sets. Thus $M / G$ satisfies the conditions of Theorem 3.3 and $M / G \cong M$.

As indicated in [8], there are numerous examples of cell-like, u.s.c. decompositions $G$ of $\sigma$-manifolds $M$ such that $M / G$ is not homeomorphic to $M$. In such cases one then is led to ask if $M / G$ is a $\sigma$-manifold factor. $M / G$ is a $\sigma$-manifold factor if there is a space $Y$ such that $(M / G) \times Y$ is a $\sigma$-manifold. If $N$ is an $n$-manifold and $H$ is a cell-like u.s.c. decomposition of $N$ such that $N / H$ is finite dimensional, then $(N / H) \times R^{5}$ is always an $(n+5)$-manifold $[6]$. The next result indicates that there is such a stabilizing factor for a large class of decompositions of $\sigma$-manifolds.

Proposition 4.2. If $M / G$ is the countable union of finite-dimensional compacta, then $(M / G) \times \sigma \cong M$.

Proof. Assume $f: R^{k} \rightarrow(M / G) \times \sigma$ and $\delta: R^{k} \rightarrow(0,1)$ are given. If $f(x)=$ $\left(f_{1}(x), f_{2}(x)\right) \in(M / G) \times \sigma$, then $f_{2}: R^{k} \rightarrow \sigma$ may be approximated by an injection $f_{2}^{\prime}$ with $f_{2}(x)$ and $f_{2}^{\prime}(x)$ being within $\delta(x)$ of each other. Now $f^{\prime}=\left(f_{1}, f_{2}^{\prime}\right)$ is one-to-one and $(M / G) \times \sigma$ satisfies the EIP. Thus if we view $(M / G) \times \sigma$ as the decomposition space $(M \times \sigma) / G^{\prime}$, where $G^{\prime}=\{g \times t: g \in G, t \in \sigma\}$, it follows 
from Proposition 4.1 that $(M / G) \times \sigma$ is a $\sigma$-manifold, and hence homeomorphic to $M$.

In the above result, the lack of information about the decomposition $G$ forced the use of a "large" stabilizing factor $\sigma$. With more information it is possible to use a finite-dimensional stabilizing factor which depends on the decomposition $G$.

Proposition 4.3. If $N_{G}=\bigcup\{g \in G: g \neq$ point $\}$ is a closed subset of $M$ with $\operatorname{dim} N_{G} \leq n$ and $M / G$ is the countable union of finite-dimensional compacta, then $(M / G) \times R^{2 n+1} \cong M$.

Proof. Again, let $f: R^{k} \rightarrow(M / G) \times R^{2 n+1}$ and $\delta: R^{k} \rightarrow(0,1)$ be given, and define

$$
f(x)=\left(f_{1}(x), f_{2}(x)\right) \in(M / G) \times R^{2 n+1} .
$$

Since the projection $\pi: M \rightarrow M / G$ is a cell-like mapping, there is a lifting $g_{1}: R^{k} \rightarrow$ $M$ of $f_{1}$ such that $d\left(\pi\left(g_{1}(x)\right), f_{1}(x)\right)<\delta(x) / 4$ [7]. Approximate $g_{1}$ by an injection $\tilde{g}_{1}$ with $d\left(\pi\left(\tilde{g}_{1}(x)\right), \pi\left(g_{1}(x)\right)\right)<\delta(x) / 4$. Define $\tilde{f}_{1}(x)=\pi\left(\tilde{g}_{1}(x)\right)$ for $x \in R^{k}$.

Since $N_{G}$ is a closed subset of $M$ with $\operatorname{dim} N_{G} \leq n,\left(\tilde{g}_{1}\right)^{-1}\left(N_{G}\right)$ is a closed subset of $R^{k}$ of dimension at most $n$. We may approximate $f_{2}: R^{k} \rightarrow R^{2 n+1}$ by a mapping $\tilde{f}_{2}$ such that $\tilde{f}_{2} \mid\left(\tilde{g}_{1}\right)^{-1}\left(N_{G}\right)$ is an injection and the distance from $f_{2}(x)$ to $\tilde{f}_{2}(x)$ is less than $\delta(x) / 2$.

Now $\tilde{f}(x)=\left(\tilde{f}_{1}(x), \tilde{f}_{2}(x)\right)$ defines an injection of $R^{k}$ in $(M / G) \times R^{2 n+1}$, so $(M / G) \times R^{2 n+1}$ is a $\sigma$-manifold by Proposition 4.1.

\section{REFERENCES}

1. F. D. Ancel, The role of countable dimensionality in the theory of cell-like relations, Trans. Amer. Math. Soc. 287 (1985), 1-40.

2. C. Bessaga and A. Pelczyński, The estimated extension theorem, homogeneons collections and skeletons, and their applications to the topological classification of linear metric spaces and convex sets, Fund. Math. 69 (1970), 153-190.

3. J. W. Cannon, The recognition problem: what is a topological manifold? Bull. Amer. Math. Soc. 84 (1978), 832-866.

4. (1979), 83-112.

5. T. A. Chapman, Dense sigma-compact subsets of infinite-dimensional manifolds, Trans. Amer. Math. Soc. 154 (1971), 399-426.

6. R. J. Daverman, Products of cell-like decompositions, Topology Appl. 11 (1980), 121-139.

7. R. D. Edwards, Approximating certain cell-like maps by homeomorphisms, Preprint. See Notices Amer. Math. Soc. 24 (1977), A-649, \#751-G5.

8. J. P. Henderson and J. J. Walsh, Examples of cell-like decompositions of the infinite-dimensional manifolds $\sigma$ and $\Sigma$, Topology Appl. 16 (1983), 143-154.

9. R. C. Lacher, Cell-like mappings and their generalizations, Bull. Amer. Math. Soc. 83 (1977), 495-552.

10. J. Mogilski, Characterizing the topology of infinite-dimensional $\sigma$-compact manifolds, Proc. Amer. Math. Soc. 92 (1984), 111-118.

11. F. Quinn, Ends of maps and applications, Ann. of Math. (2) 110 (1979), 275-331.

12. H. Torunczyk, Skeletonized sets in complete metric spaces and homeomorphisms of the Hilbert cube, Bull. Acad. Polon. Sci. Sér. Sci. Math. 18 (1970), 119-126.

13. (1980), 31-40. 
14. J. J. Walsh, Detecting finite and infinite dimensional manifolds, Address to 811th meeting of Amer. Math. Soc., April 13, 1984, Richmond, Va. See Abstracts Amer. Math. Soc. 5 (1984), 182, \#811-57-01.

15. J. West, The ambient homeomorphy of incomplete subspaces of infinite-dimensional Hilbert spaces, Pacific J. Math. 34 (1970), 257-267.

Department of Mathematics, Texas A\&M University, College Station, TEXAS 77843-3368

Current address: Department of Mathematics, Colorado College, Colorado Springs, Colorado 80903 